\title{
An Analysis of the Connection Between AI Development and the Creation of Games and Advanced Computer Systems
}

\author{
Ewa Drabik \\ Warsaw University of Technology, Warsaw, Poland
}

\begin{abstract}
Electronic games and video games have engaged the interest of artificial intelligence (AI) researchers. The cryptocurrency bitcoin is generated by an algorithm based on cryptography technology. High frequency trading (HFT) based on high quality software. The most spectacular games and programs use a learning neural network, for example backgammon, bridge, Go. Those were created with the use of "deep learning" on multiple layers of neural network. Sometimes, it is not clear "which path the computer will chose". The paper aims at discussing the electronic version of some games and their applications in developing an AI.
\end{abstract}

Keywords: AI, Go, chess, video games, cryptocurrency, neural network

\section{Introduction}

Video games are derived from board games and various sports disciplines. Their mass production began in the 1970s. Their development has impacted technological advancement, and contributed to the design of ever more sophisticated algorithms based on neural networks (see http://www.ai.c-labtech.net/sn/sneuro.html) and genetic algorithms, mostly used in developing artificial intelligence (AI).

Gaming has engaged the interest of AI researchers almost from the very beginning. The first programs were notable for incorporating mechanisms enabling them to learn from experience. A stronger version was developed for the use of "genetic algorithms" and "evolutionary" computing. The culmination was the a famous chess game in 1997, the victory of Deep Blue over defending world champion Gary Kasparov in a widely publicized series of matches. Though some AI experts disparaged Deep Blue's reliance on "brute force" computer applications (i.e., checkers, backgammon, poker, bridge, and Go). Intelligent "players" are the elements or participants in a variety of electronic games. It is very important that computers, for all their mathematical and other seemingly high-level intellectual abilities, have no emotions and feelings.

Electronic games and video games are presented in Chapter 2. Neural networks and genetic algorithms are presented in Chapter 3. Chapter 4 is concerned with games, such as Go and chess, specifically computer programs designed for playing those games with the use of AI. Chapter 5 attempts to find an answer, among others, to the question of whether the bitcoin cryptocurrency is best defined as money, commodity, or digital resource.

Ewa Drabik, professor, Faculty of Management, Warsaw University of Technology, Warsaw, Poland.

Correspondence concerning this article should be addressed to Ewa Drabik, Faculty of Management, Warsaw University of Technology, Narbutta 85, Warsaw 02-524, Poland. 


\section{Research Design}

\section{Research Subject}

The paper aims at discussing the electronic versions of games, such as chess, middling poker, backgammon, bridge, and Go and their applications in developing an AI. Software designed for playing those games, such as AlphaGo-Zero (2017) can autonomously solve complex problems, which was never possible before. This shows great progress in AI development theory. Developing other video games, perhaps not as complex as the Go program, has also significantly contributed to the advancement of many AI-related disciplines, as well as human resources management, mastering techniques of eliminating opponents, or even education.

\section{Theory and Hypotheses}

Video games derived from social games, particularly board games and sports disciplines, have become the basis for designing increasingly complex and sophisticated software not only for the purpose of entertainment, but also education and problem-solving, and most importantly, perfecting AI development techniques.

The video game industry is also a huge market (over 70 billion USD in 2017), including also e-tournaments and e-sports which provide employment for a large number of programmers, managers, and similar professionals.

\section{Video Games and the Related Market}

\section{A Short History of Video Games and Their Applications}

A video game is a game played on the screen of an electronic device, such as a tablet, smartphone, computer, or a program which enables gameplay. Such games first need to be created, using computer code, and then of course sold. Their purpose can be entertainment, education, a logic, or agility based challenge for the player. Gamers have to perform tasks, such as solving problems, eliminating opponents, and competing with AI or with other players. Video games can run on personal computers, slot machines, game consoles, smartphones, TVs, and other platforms. They are derived from board games and various sports disciplines. Games became a mass product due to increasing popularity of consoles, computers, and slot machines in the 1970s.

Generally speaking, video game development progressed along with the development of computers. They were both agility based race games (e.g., "Need for Speed" and "Civilization") and role-playing games, such as "Baldur's Gate". Other well known titles include "Prince of Persia", "Quake", "Tomb Raider", and "Legend of Zelda". The year 1999 marks the first appearance of tournament games, such as "Unreal Tournament", "Counter Strike", and "Quake III Arena". The year 2000 marks a turning point for recreational games. The market saw some mixed genre titles and experiments, like "Shogun: Total War". The 3D game environment was popularized by "Grand Theft Auto III". In 2002-2006, highly interactive strategy games became increasingly popular (e.g. "Medal of Honor" and "Half Life"). In 2007, many titles premiered which are still popular today: "Assassin's Creed", "Mass Effect", "Crisis", or "The Witcher". The years 2008-2012 were dominated by sequels; many popular titles got their new editions. The trend has shifted to independent (indie) games in 2013-2014. In that period, games were popularized by internet media (YouTube/Twitch). When it comes to online games, among the most popular were "Diablo" and "Starcraft". A huge number of games were being created for a market with too little demand, which caused many small companies to face the perspective 
of bankruptcy. In 2013, the Android system was released, which together with the growing popularity of smartphones caused another boom in video game production.

Video games have many applications. In the 1950s, they were used as training simulations for soldiers. They can also be applied to employee trainings improving competitive skills and modern management. Since the release of "Oregon Trail" in 1974, video games are also used for educational purposes. But primarily, just like the social games that precede them, they are a source of entertainment. A number of organized gaming competitions exist, such as "Poznań Game Arena" or "Intel Extreme Masters", where the number of contestants in 2016 exceeded 110,000. There are also sports tournaments. An organization overseeing e-tournaments, "Cyberathlete Professional League", was established in 1997 in Texas (USA). In the following years, other e-sports organizations appeared, such as "Turtle Entertainment GmbH", "Word Cyber Games", or "Major League Gaming". The relatively young discipline that is e-sport faces similar issues as traditional sports (i.e., corruption, doping, and insolvency). E-sport players, similarly as their "traditional" counterparts, practice several hours a day to keep their skills sharp. This includes physical workout, in order to prepare the body for the exertion of a tournament! For particular games, sparring matches are played. In electronic tournaments, it is not the nationalities and names of the players that matter, but rather team membership and the "nicknames" that the players use in-game. Around 400 million people worldwide are e-sport fans. There is growing support for the idea of including an e-sport discipline for the first time during the 2024 Olympic Games in Paris. The games included in the Olympics would have to be free of violence: strategy and agility based, card games, football, etc. Globally, e-sport is evolving rapidly. Since 2013, the finals of the international e-tournament "Intel Extreme Masters" are held in Katowice (Poland). Famous teams, such as Manchester City-Paris Saint, Germain, Ajax Amsterdam, AS Roma, and Legia Warszawa have their own e-sport teams. Competitions and tournaments are one of the reasons why video games are a rather large branch of the global market. E-sport was worth around 500 million USD in 2016, and that value increases by 100-200 million annually.

\section{Video Games as a Market Product and a Work of Art}

Video games include music, images, and narration, which can all be art forms. There are video games that are considered classics. However, to consider a video game as a work of art is a controversial notion, one that has been debated since the late 1980s. In 1989, the Museum of the Moving Image in New York had an exhibition dedicated to games, such as "Hot Circuits: A Video Arcade". This was met with a lot of criticism from many professional art critics and scientists who claimed, among other things, that video games cannot be considered art since they include a necessary element of competition and victory or failure. After all games, such as chess, Go, Pong, or Tetris can hardly be considered art. However, some claim that games featuring a plot and interesting visuals should be treated as a new genre of art and culture. Examples of such games include "Myst" (1999), "Bastion" (2011), "Ori and the Blind Forest" (2015), and "Hopy" (2017). These are usually simple adventure games or puzzle games with elaborate visuals and plot, for example, "The Talos Principle" (2014). Another reason for treating video games as an art form can be the fact that they can touch upon challenging topics, such as the meaning of life, the future, and its social consequences. Works in the video genre are typically dynamic, which brings in an element of surprise and enhances the narration.

\section{The Distribution and Sales Models of Video Games}

Polish game-makers have established their brand worldwide mainly due to titles, such as "The Witcher 3", "Earth 2140", "Dead Island", "Call for Juarez", and "Painkiller". Rapidly growing companies, such as compact 
disc (CD) Projekt, Techland, or City Interactive produce mostly games intended for smartphones, tablets, and browsers. In order to win over international markets, they create games with a high level of difficulty and long playthrough time.

In the 1980 s, the most commonplace video game storage medium was a CD, however its capacity soon proved too small. Games began to appear on Digital Video Discs (DVDs), which were an optical storage medium like the CDs, except that due to increased storage density they had larger capacity. Initially, games could only be purchased in physical copies from stores. Starting in 2003, games began to be distributed over the Internet using the Steam platform. In this way, game producer could save money on DVDs, boxes, logistics etc. Some games are also available for free on the Internet; however, they usually include in-game content that is only made available after payment. It is also possible to play games for free in early access. This is a strategy of making games available at the early production stage. Money gained from early access is then used to further develop the game.

\section{Europe Game Classification System}

Pan European Game Information (PEGI) is a game classification system used in all of Europe. The tags provide information ensuring that the entertainment products, such as movies, videos, or games are appropriate for a given audience. For example, a game tagged as PEGI7 is appropriate for seven-year-old children, while a PEGI18 game is intended for adult audiences only. The PEGI tags also consider the themes included in the game. In order to obtain certification, a game distributor must prior to release fill out a questionnaire in which she outlines 37 points pertaining to the anticipated reactions of the users, as well assure that the game does not promote violence. Moreover, the game itself, along with the promotional materials, must be presented to the PEGI committee.

\section{The Video Game Industry and Its Workforce}

The video game industry employs traders, managers, marketing experts, programmers, beta testers, and game designers. A game designer is essentially a programmer who, in addition to coding skills, has extensive general knowledge in many fields. This is due to the fact that game-making requires a lot of interdisciplinary knowledge. For instance, graphics expertise is a must and this goes beyond simple computer visuals: One must know how lighting changes colors, be familiar with the physics of the Doppler effect, wave interference, shortest path algorithms, and even storytelling. An average game's source code is ca. 200-300k lines of code. Programmers are divided into specialized teams responsible for designing 3D models, graphics, screenplay etc. The creative process behind a game is so challenging that it can be safely claimed that games are particularly complex systems which share certain features associated with AI. Some of them, particularly interactive ones, such as Go and chess, have significantly contributed to the advancement of AI.

Due to their huge complexity, all games should be examined by testers. No large chunk of code is free of errors; they are virtually impossible to avoid. Contrary to popular opinion, a job that involves playing games all day long is not quite as pleasant as one might think. The work is very repetitive, and it requires a high level of concentration and attention to detail. A tester's job does not end at spotting errors. They also carry out black-box tests, which involve investigating what elements present a difficulty to players.

Another unavoidable part of game-making is the so-called "crunch phase". The term refers to the final stages of game production and the necessity to meet deadlines. Some companies introduce the role of line 
manager, who is responsible for organizing the time, space and proper working conditions for programmers and testers alike.

\section{Risks Associated With Games}

It is not without reason that video games are divided into appropriate age categories: Some titles are safer to play for children and the elderly. There is a concern that gaming can cause increased aggression and often also addiction in the players. In December 2017, the World Health Organization (WHO) declared that the addiction to video games should be considered a mental illness. However, some psychiatrists specializing in addictions claim that video game addiction is a result of environmental factors rather than the games themselves. The risk of addiction is highest in people who have a tendency to lose themselves in a virtual world. Gamers are mostly young people, as shown in Table 1. The table shows the percentage of each age group within the gamer population.

Table 1

Gamers Age Groups in 2013 and 2015

\begin{tabular}{lll}
\hline Age group & $2013(\%)$ & $2015(\%)$ \\
\hline $15-24$ years old & 37 & 43 \\
$25-34$ years old & 33 & 32 \\
$35-44$ years old & 24 & 16 \\
$45-55$ years old & 5 & 9 \\
\hline
\end{tabular}

Source: Cholerzyński (2017).

Most gamers are young people. Table 1 shows an increase in gamers above 45 years old, as well as those aged 15-24.

When it comes to the regions of the globe which are "dominated" by gamers, the largest gamer population and biggest turnover is associated with Asia and the Pacific. This might be a cause of global trends of rapid economic growth in Far East countries, China in particular. However, the largest increase in video game sales was observed in Latin America, as shown in Table 2.

Table 2

Turnover in USD on the Video Game Markets in (Particular) Regions of the Globe and Growth Compared to Preceding Year

\begin{tabular}{lll}
\hline Region & Market value & Annual growth (\%) \\
\hline Europe, Far East & 23.5 billion USD & 7.3 \\
South America & 4.1 billion USD & 20.1 \\
North America & 25.4 billion USD & 4.1 \\
Asia and the Pacific & 46.6 billion USD & 10.7 \\
\hline
\end{tabular}

Source: Newzoo Free (2016).

In 2016, the global game market was worth ca. 100 billion USD, with a growth of $8.5 \%$ compared to the previous year. It is undoubtedly a growing market. A superiority, weakness, opportunity, and threats (SWOT) analysis of the market suggests that its strengths are: genre variety, varied sales, distribution models, and the possibility of crowdfunding (financing the project by receiving donations from subscribers). Its weaknesses are: complex gaming jargon, the trend of viewing gaming in a negative light, concerns about health hazards, high difficulty for small game producers to succeed on the market, the uncertainty related to the sales, and reception 
of a game. The opportunities are: the growing popularity of games, internet media popularizing the industry, an increase in popularity of social games, and growing diversity in price range. The main threats are: piracy, high competition when it comes to free-to-play games, customs barriers, and currently relatively low popularity of e-sport and other gaming events (Cholerzyński, 2017). A significant advancement in the production of games, particularly those based on board games, was due to the AI research.

\section{AI, Genetic Algorithms, Neural Networks}

\section{Basic AI Terminology}

Information processing in computers and similar devices occurs on appropriate representations of data. These can be letters, numbers, writing, images, electromagnetic signals, sound, signal chains, etc.

"Coding" refers to the process of assigning information to data according to established rules. A piece of information is assigned a corresponding string of characters (signals). The rules of assigning information are called a code. The same piece of information can be expressed using different data (i.e., represented by different code). A crucial role in information processing is played by algorithms.

- "Algorithms" are ordered strings of operations such that by following the steps, a specific goal will be achieved (e.g., solving a mathematical problem or a technical issue).

- "Continuous operations (analog)" are calculus, arithmetic operations on functions.

- "Discrete operations (digital)" are arithmetic and logical operations.

Every task can be described using bits of information (i.e., strings of ones and zeroes). When it was observed that any thought process can be described using ones and zeroes, the science of AI was established. The term was coined by John McCarty ca. 1955, defined as follows: AI is the science and engineering of making intelligent machines.

Currently, AI is considered a science dedicated to creating and studying intelligent behavior algorithms. It includes these of the programming methods which cause computer behavior similar to human thought. On the other hand, it is understood that human intelligence is the ability to recognize observed reality, to understand and establish goals, and seek means to achieve them.

The primary goal of AI is to accumulate knowledge (i.e., learn). Other major problems in AI are:

(1) Decision making in conditions of incomplete data;

(2) The analysis and synthesis of natural languages;

(3) Logic based games such as chess and Go;

(4) Expert and diagnostic systems.

The technologies used in industrial plants, which function in conditions of incomplete data, are also based on the same principles as AI. Automatic text translation is not on par with humans, but Google Translate is a constantly improving tool designed for this purpose. Neural networks have improved the functioning of processes, such as speech recognition, following verbal commands, and answering to queries. Algorithms make it possible to analyze images, stylize any photograph to look like the work of Van Gogh, Picasso on Munch, generate short poetic forms, compose, etc. Self-learning algorithms allow better care for health or value of provided services. The knowledge gathering process associated with AI is based on data exploration. For this purpose, both genetic algorithms and neural networks are used. AI is increasingly used in the power industry, in the creation of systems monitoring specific processes, in planning, and in decision-making. It is also used for image processing (e.g., in cameras), financial decisions support, and many other areas of everyday life. In 
economy, there is a widespread use of expert systems that automatically assess (e.g., creditworthiness or best client profile) as well as plan marketing campaigns.

\section{Genetic Algorithms}

The theory of genetic algorithms was established based on analogical processes observed in natural evolution. Due to the mechanism of natural evolution, living organisms have reached an incredible level of complexity. Evolution occurs in chromosomes (i.e., genetic chains containing coded information about the given specimen). An important part of evolution is natural selection, which ensures that the only surviving organisms are those which increase the chances of processing and reproduction in a competitive environment. Evolution is based on the exchange of genetic material and on mutation.

The classic genetic algorithm K-AG operates on sets of zero-one chains. A single element of a chain is called a gene, while a whole chain is a chromosome. A set of chromosomes is called a population.

Outline of the K-AG algorithm is as follows:

(1) The starting population mechanism is based on the random formation of the desired number of chromosomes (coin toss, random number generator).

(2) The mechanism of quality assessment for a given chromosome is based on calculating the value of the fitness function. The purpose of this function is to state to what degree the chromosome can solve a problem.

(3) Chromosome selection is a mechanism based on selecting the chromosomes that will become the parents of next generations. The selection should proceed in such a way that the parent chromosomes have the highest possible value of the fitness function. The selection itself is random; however, the selection methods should be chosen in such a way that the high fitness chromosomes have the highest chances of being selected.

(4) The mechanism of mutation occurs on a single chromosome and changes its value.

(5) The crossover of chromosome pairs for the purpose of exchanging genetic material between chromosomes to create new specimens. This should create an improved new generation. The new generation may completely replace the old, from which only the lowest fitness specimens will be removed.

(6) Establishing a new generation. The newly generated children may: completely replace the old generation, replace its parents, and join the old generation with the exclusion of several (randomly selected) specimens.

(7) Assessment of the new generation based on the fitness function.

Major applications of genetic algorithms are:

- optimization of database queries;

- stock market value forecasts;

- extracting information from data;

- creating models of population migration;

- creating memory portraits etc.

Apart from genetic algorithms, another crucial role in creating an AI is played by neural networks.

\section{Artificial Neural Networks}

A neural network is an information processing system which utilizes the biological models of nervous systems. The history of neural networks begins in the mid-20th century. One of the first steps in the field was taken by Ada Byron (1815-1852) who built a primitive calculating machine and claimed to see no reason why machines should not be able to learn. It can be said that neural networks came into existence after the 
publication of "A logical calculations of the ideas immanent in nervous activity" by McCulloch-Pitts in the Bulletin of Mathematical Biophysics in 1943. It is a model of binary inputs with a $w_{i j}(i, j=1, n)$ weight, with excitability threshold $b_{i}$, whose purpose is to determine what value of the weighted sum of the inputs should be achieved or exceeded. The neurons form a network which has the ability to learn. There are cellular, feedforward, multilayer, and recurrent networks. A cellular network is one in which each neuron is connected to the adjacent neurons. Feedforward networks are usually composed of several layers (see Figure 1); there are connections between neurons on different layers, but neurons on the same layer are not connected to each other.

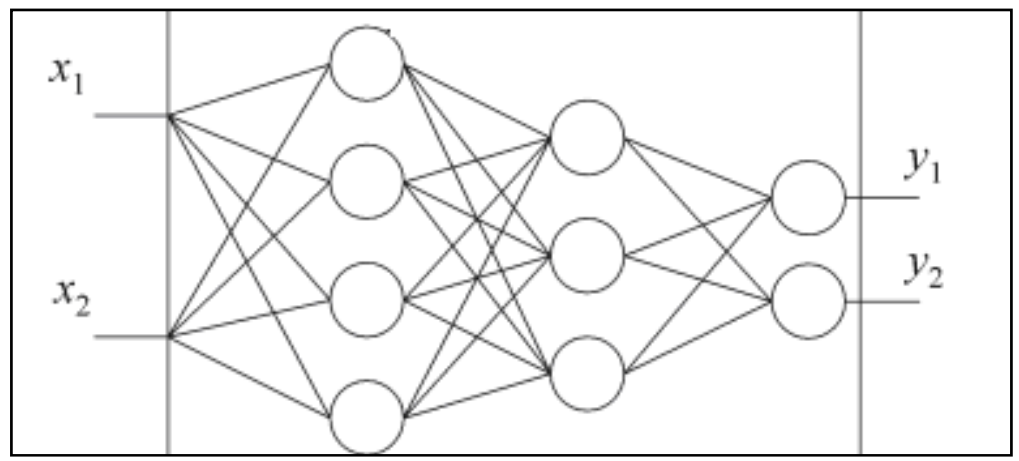

Figure 1. Visual representation of a multilayer neural network. Source: Own design based on illustration submitted to a website http://www.ai.c-labtech.net/sn/sneuro.html.

Figure 1 depicts an example multilayer neural network. A recurrent network is one in which part of the output signals are at the same time input signals. A network graph node corresponds to a single neuron. Its direction is from one neuron's output to another neuron's input. A layered neural network consists of: an input layer, hidden layers, and an output layer. The neurons are placed on layers so that there are no connections between neurons on the same layer. There are only connections between neurons placed on neighboring layers. The principle of a neuron's operation is that a weighted sum of input values is used to measure excitability. The following designations are used:

$$
\begin{aligned}
& \mathrm{X}=\left[x_{1}, x_{2}, \ldots, x_{n}\right] \text { learning vector, } \\
& \mathrm{Y}=\left[y_{1}, y_{2}, \ldots, y_{M}\right] \text { output vector, } \\
& \mathrm{D}=\left[d_{1}, d_{2}, \ldots, d_{M}\right] \text { desired response vector, } \\
& W=\left[w_{1}, w_{2}, \ldots, w_{n}\right] \text { weight vector. }
\end{aligned}
$$

Excitability is calculated in the following way:

$$
h_{i}=\sum_{j=1}^{n} w_{i j} x_{j}+w_{i 0}
$$

where $w_{00}=1$.

The output is created as a result of applying excitability to the output function, also called the activation function: $y_{i}=g\left(y_{i}\right)$,

The learning method of feedforward networks is the optimization of the goal function.

$$
E(W)=\frac{1}{2} \sum_{k=1}^{M}\left(y_{k}-d_{k}\right)^{2}
$$


where $M$ designates the number of neurons on the layer.

One of the methods of error minimization (2) in a neural network is to modify the weights (e.g., according to a growth algorithm and adjust them as necessary). In other words, the network "learns" by adjusting the weight values $w_{i j}$ in iterations. The task of a training set is to present the network with cases that it might encounter during operation. All case classes should be represented in the given model of a neural network. A testing set is used to verify the network during and after the learning process. Throughout the learning process, the mean squared error should decrease. The learning procedure consists of modifying each neuron's weight based on the error. If the goal is $d$ and the actual result $y$, then the error is measured as $(d-y)^{2}$. The total error value for the whole network is calculated. The aim of the procedure is to adjust the weights to achieve the largest possible decrease in error. Neuron weights are modified, starting with the output neurons. This is called a backward propagation method. The weights can also be optimized using genetic algorithms. In this case, the weights are coded as a binary string (chromosome) and weight optimization is achieved by establishing a weight vector for which the fitness function (defined as a sum of squared errors) is the lowest. We differentiate between supervised learning, for example, the network compares the output with the known correct answer $d_{i}$, (return signal contains information about whether or not the output is correct), and unsupervised learning wherein the only available information is the correlations between input data. The network establishes categories based on those correlations and generates signals corresponding to specific categories. The following example illustrates in what way the weights are assigned.

Example. Input data consist of numbers. If input signals are 1, 1, 0, then the input vector is: $[1,1,0] \mathrm{T}$.

An artificial neuron assigns to each channel a separate number-a weight: [2.5, -1, 0.5].

A number is generated based on the signal and the weights:

$[1,1,0]$ T. $[2.5,-1,0.5]=2.5-1=1.5$

The output information is dependent on the total excitability value.

The procedure consists of generating 1 if it does not exceed a predefined threshold.

E.g., if excitability is 1.5 , the neuron will generate 1 .

Another basis for network learning is the dynamic interactions based on observing individual states, and implementing a strategy after which the network may receive a positive reinforcement (reward). The goal is to select a strategy in a way that maximizes the reward-defined metric of quality. The major applications of reinforcement learning are:

- ooptimal control;

- construction of self-learning robots;

- board games.

The most spectacular examples include using a learning neural network for playing trik-trak (backgammon) and Go. As a result of playing many games "against itself", the network achieves mastery.

Designing a neural network consists of establishing its topology (i.e., setting the number of neurons on layers and the number of layers in the network). The next steps are: the preparation of a training set and a testing set, network learning, and verification of the results. Networks are usually approximators of a continuous function. They transform an $n$-dimensional set of input data into an $M$-dimensional set of output data.

It should be mentioned that to achieve text recognition with a neural network, one should use an alphanumeric code: $1 \rightarrow \mathrm{A}, 2 \rightarrow \mathrm{B}, 3 \rightarrow \mathrm{C}$...Both genetic algorithms and neural networks were used to beat 
chess masters and Go master alike.

\section{Chess, Go, and AI}

Chess has served as a model for the creators of AI and has contributed to the development of computers with ever increasing computing power. Beginnings are often hard and such was the case with creating chess based programs (Drabik, 2017). In 1993, a computer "won" a match with the young chess master Judit Polgar. Another breakthrough turned out to be the multiprocessor computer called Deep Blue, which was able to use specialized chess processes to generate a position, evaluate its value, and analyze further moves. In February 1996 in Philadelphia, the computer lost in its first duel with Gari Kasparov. However, in May 1997, in New York, it managed to beat him. The fact is that its advantage was in enormous computing power and the ability to quickly access an extensive database. For the analysis of chess positions, it used a procedure called selective follow-up (alternative moves were investigated in a loop; if the position was judged to have a lower value the operation was interrupted and the program selected the move that it rated as having the highest value). Some computer technologies were based on Deep Blue's experience, for example, Distributed Resource Management System (DRMS)-dynamic management of parallel environments and United Trace Environment (UTE) - universal rules for software running in parallel mode. In turn, parallel processing technologies are used in molecular dynamics, seismic analysis, meteorology, and business intelligence which include data mining, decision support, and knowledge management. In 2011, International Business Machine (IBM) also developed an intelligent computer capable of understanding human speech, called Watson. The machine can gather and search through a huge amount of information. It has won, among others, a duel with two champions of the American "Leopards" tournament. Now, a new generation of computers is being developed-quantum computers (i.e., ones that utilize a certain class of quantum phenomena). Those machines will be capable of autonomous decision making.

Go is one of the oldest games in the history of mankind. It was created in China in the years 2357-2255 BC. Go is a positional combinatorial game with zero-sum information. Go is a strategy game. The elements necessary for the game are: a square board with a grid of 19 intersecting lines and 360 pieces (called "stones"): 180 black and 180 white (Drabik, 2017). Go has contributed to the development of computer programs and AI. In 2016, a computer called Deep Mind using the AlphaGo program defeated a Go world champion, Lee Sedola from Korea (score 4:1). Deep Mind, after playing many games (in other words, after numerous optimizations) made a connection between bouncing a stone and collecting points.

In the recent years, AlphaGo was modified. This was done with the use of "deep learning" (unsupervised) on multiple layers of the neural network. The process is not pre-determined. Therefore, only the results can be observed; it is not clear "which path the computer will choose". This method was used to create AlphaGo-Zero, which beat its predecessor, AlphaGo, in 2017. AlphaGo-Zero could learn from its mistakes. It can therefore be assumed that it has passed the cognitive threshold of a human mind! It may turn out that we have a self-aware machine which could lead humanity to new discoveries, or give humans eternal life. Consciousness is a particular mental condition which can be achieved. However, such an evolution would require thousands of years. For now, we can only regret the fact that AlphaGo-Zero has no notion of being a world champion.

\section{Games on the Capital Market}

The cryptocurrency bitcoin is an Internet-based means of payment independent of any country's central 
bank. Cryptocurrency can be generated by anyone with a network presence and access to significant computing power. New bitcoins are generated by an algorithm based on cryptography technology. The system presents a potential bitcoin buyer with a mathematical problem which she must solve! Solving the problem is not just a matter of skill, but also a fast machine. On average worldwide one bitcoin is generated every 10 minutes. The Chinese in particular are very proficient in their "production". Every bitcoin is unique, its authenticity verified by the network. By the end of 2017 there were 16 million bitcoins. In theory, their production is to stop at 21 million.

The market price of a bitcoin is so high that we can assume that there is a speculative bubble emerging. This threatens with a crisis-not financial, but an energy crisis. The broadcast and maintenance of the system which supports the cryptocurrency consumes huge amounts of energy. A chance for cryptocurrencies lies with effectively "thinking" algorithms with some AI components. The managers of cryptocurrencies have a very extensive, multilayered marketing system. They are also well-versed in psycho-economy. They make covert offers inviting one to engage in a network of affiliated marketing (i.e., ads exchange system). They skillfully use certain psychological manipulation tools in order to entice potential gamers, who have a tendency towards "magical thinking". This is accompanied by what is known as "confirmation bias": the selective choice and interpretation of information so that it confirms the predefined expectations. The gamers are often confident in their own skill.

Binary options are another game generating a speculative bubble. It is a type of market hazard game where the aim is to bet on the behavior of the exchange rate of a currency, resource, stock, stock market index etc. in the immediate future (minutes or even seconds). It is also possible to bet on whether the rate will reach a certain limit. In this case the opponent is the broker-option issuer.

Similar mechanism is behind Forex (Foreign Exchange), an international currency exchange market, based on financial leverage. A trader works from her computer, buying and selling contracts for currencies or other financial instruments. An agreement assures that on the day when the contract is finalized the seller will pay the difference between the current value of the asset and its value on the day the contract was signed. If the difference is negative, it is the buyer who pays. There is a certain risk associated with the transactions and most of the information available is on the seller's side. Thus the buyer is in danger of falling victim to the seller's abuse, which is a classic example of a so-called moral hazard.

Playing on the capital market can result in huge losses for an independent investor. Transactions on a gigantic scale occur on the markets, mostly through complex computer systems. Any change on the market can cause rapid sales of financial instruments and a huge flow of capital, and the rules of the "game" are never symmetrical. Specially programmed, very fast computers generate more than a half of all transactions on the global markets. There were incidents (such as the 6th of May 2010) when stock prices begin falling rapidly and a billion dollars disappears from the market. After 20 minutes, everything is back to normal and most of the losses are recovered. The blame for these sudden price changes (flash crashes) is put on software. However, there is also a suspicion that the initial price drops were caused by an investor (or several) placing a large order. The computers have misinterpreted this as a sell-out signal and caused a temporary suspension of stock quotes and markdowns. This event has been recorded in the capital market history.

Computers take into account a great number of variables during decision making, including technical analysis results. A human can never keep up with all the data that needs to be analyzed. This is called high 
frequency trading (HFT). The strategy is to make as many transactions as possible in a short time (e.g., in order to decrease the difference between purchasing price and selling price). Psychologist Daniel Kahneman, winner of Nobel Prize in economics, has pointed out that humans lose to machines ever more often.

\section{Conclusions}

The use of neural networks for video game production has become a breakthrough in AI research. Already in 1997, when Kasparov was losing a chess match against Deep Blue, he was competing against "the spirits of his predecessors" (chess masters). However, it was only in 2017 that the program AlphaGo-Zero has shown that a machine which can think independently thanks to neural networks is the true world champion at Go. The processing in networks occurs on many paths simultaneously, which means many operations can be carried out at once. It is however not the speed and processing power that guarantee that a computer will prevail over humans. An important advantage of neural networks is that they can discover patterns in data sets which for a human would be unclear or even invisible. Neural networks are highly adaptable, since unlike humans they can use data which is corrupt or even incorrect. They know how to approach data they've never encountered before. This is why they are being more and more widely used (e.g., to classify objects and images, reduce interference or make predictions in both methodological and economic modeling). Similarly, HFT based on high quality software is the future of the world economy. The asymmetry in qualities, such as processing speed spells failure to the future investors who would attempt to compete against the machine.

\section{References}

Bobrowski, N., Rodzińska, P., \& Socha, M. (2015). The condition of Polish line video games. Retrieved from http:/www/kpt.krakow.pl/wp-content/uploads/2015//09/Raport A4 Web.pdf

Bomba, R. (2012). Gamification. What the reality stay cyber game? In A. Pitrus (Ed.), The giant in shade: The video games in audio culture (pp. 67-90). Cracow: Jagiellonian University Publishing House.

Cholerzyński, R. (2017). An analysis and recommendation on the video games market in Poland (B.A. dissertation, Warsaw University of Technology).

Drabik, E. (2016). Several remarks on the role of certain positional and social games in creation of the selected statistical and economic applications. Foundations of Management, 8(1), 289-296.

Drabik, E. (2017). Reverse engineering, some board games as a key to the development of artificial intelligence and construction of economic models. Economics Word, 6(2), 121-132.

Kwiatkowska, A. M. (2007). The system of form further of decisions. Warsaw: PWN.

Newzoo Free. (2016). Global games market report. Retrieved from http://resources.newzoo.com/hubs/Reports/Newzoo Free 2016/

Ostrowski, K. (2016). The short stories of e-sport. Retrieved from http://www.gram.pl/artykul/2016/02/09/krotka-historia-e-sportu -czyli-od-czego-sie-wszystko-zaczelo.shtml

Sieja, B. (2013, July). The history about game (2nd ed.). CD-Action, 92-93. 